\title{
On the mechanism of catalysis induced by mechano-activation of solid body
}

\author{
ANDRZEJ KULCZYCKI ${ }^{1 *}$, CZESŁAW K. KAJDAS ${ }^{2}$, HONG LIANG $^{3}$ \\ ${ }^{1}$ Air Force Institute of Technology, Warsaw, Poland \\ ${ }^{2}$ Warsaw University of Technology, Institute of Chemistry in Plock, Poland \\ ${ }^{3}$ Department of Mechanical Engineering, Texas A\&M University, USA
}

\begin{abstract}
The paper presents a new model of the mechanism of mechanocatalysis and tribocatalysis. The reason for the increase in heterogeneous catalysis effect after mechanical activation of a catalyst has not been fully understood yet. There is no known theory, which would explain the mechanism of the influence of mechanical energy introduced to catalyst particles on the rate of chemical reaction. All existing theories are based on Arrhenius equation and assume that catalysts increase reaction rate due to decreasing of activation energy $E_{a}$. We hypothesize that both for standard and catalyzed heterogeneous reactions the same $E_{a}$ (real activation energy) is needed to trigger the reaction processes and the catalytic effect is the result of energy introduced to the reaction system, its accumulation by a catalyst and then emission of high flux of energy to the space near the catalyst particles. This energy emitted by molecules of reagents can reach a value equal to the value of $E_{a}$ at lower ambient temperature than it would result from Arrhenius equation. This hypothesis is based on $\alpha_{i}$ model described in previous papers by Kajdas and Kulczycki as well as the results of tribochemical research described by Hong Liang et al., which demonstrate that the reaction rate is higher than that resulting from temperature.
\end{abstract}

Keywords: tribochemistry; activation energy; catalysis

(C) Wroclaw University of Technology.

\section{Introduction}

Tribochemistry and mechanochemistry are the coupling of mechanical and chemical phenomena on an atomic and molecular scale and include mechanical breakage, chemical behavior of mechanically-stressed solids (e.g. stress-corrosion cracking), tribology, polymer degradation under shear, cavitation-related phenomena (e.g. sonochemistry and sonoluminescence), shockwave chemistry and physics, and even the burgeoning field of molecular machines. Mechanochemistry can be seen as an interface between chemistry and mechanical engineering. A new method has been proposed recently in order to measure the energy involved during mechanical transformations. Displacement reactions between a metal oxide and a more reactive metal can be induced by ball milling. In some cases the reaction progresses gradually

\footnotetext{
*E-mail: andrzej.kulczycki@itwl.pl
}

and a metal/metal-oxide nanocomposite is formed. Ball milling may also initiate a self propagating combustive reaction. The information available about these processes is reviewed. It is argued that the gradual or combustive nature of the reaction depends on thermodynamic parameters, the microstructure of the reaction mixture, and the way they develop during the milling process [1-4].

The unsatisfactory state-of-the-art in the theories of phase transformations and fracture is the reason of the current (and future) study. To make some progress in understanding the existing phenomena, Grinfeld [4] proposed exploring the strong features and weaknesses of the energetic (thermodynamic) methods by performing theoretical and experimental studies on macroscopic adhesive systems.

In one of our recent studies, an investigation was conducted to validate Arrhenius-Eyring equation for an in situ mechanically simulated system. It was found that the applied mechanical energy 
overrides the theoretical activation energy resulting from tribo-chemical reaction. An equation similar to Marcus equation was proposed [28].

Using this equation we studied oxidation of tantalum (Ta) under mechanical stimuli, friction. Without any doubts it was found that it was energetically possible for non-equilibrium tantalum oxides. Other approaches cannot predict processes involving friction. In addition, the phase transformation between suboxides of tantalum is reversible $[5,6]$.

\section{Theory of the mechanism of me- chanically activated reactions based} on $\alpha_{i}$ model and its application to tribocatalysis and heterogeneous catalysis

The term known as $\alpha_{i}$ is very specific. It assumes the forms that suggest the accompanied triboreactions would follow thermodynamics paths, typical of irreversible processes [7]. The relationship between $\mathrm{E}_{a}$ and enthalpy in solids is also of importance in this approach, because the energy required for the reactants in a fluid phase to go into transition state decreases, thereby decreasing the energy required to initiate the tribocatalyzed reaction.

The detailed mechanism of forming a protective lubricant layer in a space near a solid material during a tribological process was analyzed using the $\alpha_{i}$ method. The principle of the $\alpha_{i}$ method (model) was based on current knowledge about the mechanism of anti-wear (AW) and EP protective layers formation by lubricants containing additives. These tribological issues were presented and discussed by Kulczycki [8-10].

\section{Physical interpretation of $\alpha_{i}$ parameter}

$\alpha_{i}$ is a measure of the impact of an initial state of a reaction system to the maximum value of the stream of energy introduced into the system in a mechanical way; it means that if the value of mechanical energy stream is higher than this maximum value, the process cannot take place.

The value of $\alpha_{i}$ is dependent on the chemical structure of the reactants, the environment, in which the reaction occurs (a solvent, for example) and the nature of the process.

A difference between the value of $\alpha_{i}$ at the beginning of the process and at the end of this process does not depend on the way it is performed and $\alpha_{i}$ is a function of state of the system:

$$
\begin{gathered}
\alpha_{i}=[f(b)-f(a)] C \\
C=C(y) ; C=\text { const } .
\end{gathered}
$$

In this case, $\alpha_{i}$ is a linear function of $y=b$ and the parameters of this dependence are different for different functions. The relation between the values $b$ and $a$ determines the character of proportionality. In case of $b>a, \alpha_{i}$ is proportional to $y$, and, in opposite case of $b<a, \alpha_{i}$ is inversely proportional to $y$.

For different reagents, the value of $b$ is only one variable (assumption of Cauchy theorem) and a is a constant. Variable $b$ is related to tribological process conditions: for example an applied load $\mathrm{P}$.

As in tribological tests only one parameter is usually used for evaluation of lubricating additives effectiveness and this parameter characterizes the critical test conditions, resulting in seizure or weld; this parameter can be treated as variable $y$ in above equations. Variable $\alpha_{i}$ is related to a lubricant/additive as a reagent in triboreations, which occur during a tribological process. It makes possible a quantitative description of the lubricant/additive reactivity under conditions of a tribological process. The model assumes that $\mathrm{f}(y)$ relates to the energy introduced to the tribological system, and $\phi(y)$ relates to the additive/reactant reaction initiated by the introduced energy. Consequently $\alpha_{i}$ is the relative (related to $\mathrm{f}(a)$ and $\phi(a)$ ) measure of the additive/reactant ability to trigger the chemical reaction process.

The relationship between these two functions makes it possible to relate a test result to the mechanism of this tribological process. The 
same mechanism can take place during different tests. $\alpha_{i}$ is a non dimensional parameter of a value associated with lubricant properties related to a given mechanism of a tribological process/tribochemical reaction. Thus, it can be concluded that lubricants/reagents ability to create boundary layer/given reaction products may be expressed by this coefficient $\alpha_{i}$. Functions $\mathrm{f}(y)$ and $\phi(y)$ should be defined and described using the parameters measured during the test. In tribological tests y is mainly an applied load, P. For a number of lubricants, a linear dependence between $\alpha_{i}$ and y was found. In this case, equation 2 can be described as:

$$
C=[1 / \phi(b)-\phi(a)] \phi^{\prime}(b) / f^{\prime}(b)
$$

where $\mathrm{C}$ is a function of an applied load $\mathrm{P}$ and for different lubricating additives/reagents its value is constant for a number of tested lubricants undergoing the same mechanism of tribological process. Concluding, $\mathrm{C}$ is a function of $y$ and a constant value of $\mathrm{C}$ for various values of $y=\mathrm{P}$ (critical value of applied load) requires $\mathrm{C}$ to be a harmonic function of $y=\mathrm{P}$, described by the following equation:

$$
C=B \exp \left(-k_{1} y\right) \cos \left(k_{2} y+k_{3}\right)
$$

where $\mathrm{y}=\mathrm{P}$ or $\mathrm{T}$ and $\mathrm{B}, \mathrm{k}_{1}, \mathrm{k}_{2}$ and $\mathrm{k}_{3}$ are constant values.

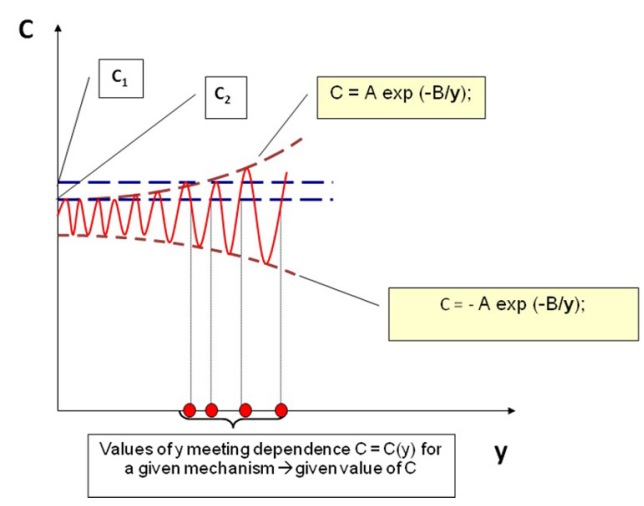

Fig. 1. Dependence between C and applied load P.

Consequently, for a given constant value of $\mathrm{C}$ only some of values $\mathrm{P}$ meet equation 4 (red points in Fig. 1). What is physical and chemical meaning of those dependences? The answer to this question seems to be also a key to tribocatalysis and heterogeneous catalysis.

\subsection{Exponential part}

Exponential part of this dependence can be connected with kinetics of chemical reactions of additive/reactant by Arrhenius equation:

$$
k=A \exp \left(B_{k}-E_{a} / R T\right)
$$

where temperature $\mathrm{T}$ may be connected with a load $\mathrm{P}$ applied in a tribological process:

$$
T=T_{b}+\mu P^{0.5} D
$$

with

$$
D=10^{-5} V_{s} P_{p}^{0.5}\left(\kappa_{1}+\kappa_{2}\right)^{-1}
$$

where: $\mathrm{V}_{s}$ - sliding speed, $\mathrm{P}_{p}$ - unit pressure of the metal flow, $\kappa_{1}$ and $\kappa_{2}$ - coefficients of thermal conductivity of cooperating elements of a tribological system.

$\mathrm{E}_{a}$ cannot be a function of the applied load $\mathrm{P}$, which is the only one variable in equations 1 and 3 . Analysis of the described above model leads to the conclusion that $\mathrm{E}_{a}$ value is constant for different additives, which give different values of critical load $\mathrm{P}$ in a given tribological process (given mechanism of protective layer formation).

The process of protective layer formation, already known and described, assumes adsorption and chemical reactions of an additive inside the lubricant layer and between the additive or product of its reaction inside the lubricant layer and on the lubricated surface. Conventional models assume that additives with different chemical structures undergo different reactions, each one of different $\mathrm{E}_{a}$.

Conclusions resulting from the $\alpha_{i}$ model are that a part of additives undergoes similar reactions during a tribological process and the resultant value of $\mathrm{E}_{a}$ for these reactions in this process is the same and triboreaction rate constant depends on load $\mathrm{P}$, equation 8:

$$
k=A \exp \left[B_{k}-E_{a} / R\left(T_{b}+\mu P^{0.5} D\right)\right]
$$

\subsection{Trigonometric part}

Trigonometric part of equation 4 can be connected with emission of electrons/photons by a 
metal/solid body surface. The energy stream is different at various angles and can be expressed as follows:

$$
e_{\gamma}=e_{0} \cos \gamma
$$

where $\gamma$ is a function of $\mathrm{P}, \mathrm{e}_{0}$ is density of energy stream in perpendicular direction to the solid body surface. The anisotropic electron emission was observed for cathodes controlled by temperature [11-14]. Since temperature in friction space is a function of applied load (equation 6), the following hypothesis was formulated: anisotropy of electron/photon emission during a tribological process depends on applied load $\mathrm{P}$, which is only one variable in the $\alpha_{i}$ model (other parameters, such as friction coefficient, should be constant). The influence of applied load, and consequently generated temperature, on anisotropy was partly confirmed by Hrach research results $[13,14]$. Anisotropy depends on the type of solid and physical and chemical structure of its surface.

This conclusion results from mathematical assumptions of the $\alpha_{i}$ model, which requires only one variable: in the model this variable is the applied load P. It was experimentally found that in 4-ball tests under conditions of critical state of protective layer (e.g. seizure) the value of friction coefficient is the same for different lubricants. Consequently, variables in equation 4 ( $\mathrm{T}$ and angle $\gamma$ ) should be functions of the applied critical load P. The relationship of temperature in friction space, $\mathrm{T}$, can be connected with $\mathrm{P}$ by equation 6 . There is no general relationship between $\mathrm{P}$ and angle $\gamma$. This physical meaning of exponential and trigonometric parts of equation 4 ( $\mathrm{C}$ value) can be expressed by the following dependence:

$$
C=B \exp \left(-k_{1} E_{a} / P^{0.5}\right) \cos \left(k_{2} P+k_{3}\right)
$$

On the basis of this mathematical model, physical model of tribocatalysis/catalysis was created. The model assumes that mechanical work done on a system (containing liquid reagents/lubricants) is transformed to internal energy, which increases its value, and dissipated energy. Internal energy is distributed in the system: one part is distributed to the liquid phase and is responsible for temperature increase of the lubricant $(\mathrm{T})$, the second part concerns/enhances the energy of electrons/photons emitted to the liquid phase from the solid surface. This part of energy is cumulated in the solid body. The energy cumulated in the solid body is emitted as electrons or photons from its surface in a form of impulses of high intensity. The energy cumulated in the liquid phase is not sufficient to reach the value of $E_{a}$. Energy emitted from the surface as impulses can reach value of $\mathrm{E}_{a}$ and reaction/triboreaction begins to proceed.

The energy cumulated in the solid body had been emitted as electrons or photons from its surface in a form of impulses of high intensity.

The value of the angle $\gamma$ depends on the system energy flux. The critical state of a tribological system appears at conditions resulting in destruction of the protective film. It has been observed that for different reactants the critical rate of reaction leading to protective layer destruction is achieved for different values of energy flux in the system (different values of applied load P). The same value of $\mathrm{C}$ obtained for different reactants and different densities of energy streams introduced into the system (characteristic for each reactant) leads to the conclusion that the same critical rate of destruction reaction has been achieved and thus, for each reactant, a different angle $\gamma$ is connected with the critical rate of reaction (for different reactants, protective layer destruction appears under different applied loads, $\mathrm{P}$, and angle $\gamma$ is characteristic of the applied load P).

Consequently, for each reactant there is a specific value of the energy flux density $\left(\mathrm{e}_{\gamma}\right)$ emitted by the solid/catalyst and the value of activation energy $\mathrm{E}_{a}$ is constant.

\section{Thermodynamic interpretation of dependences resulting from $\alpha_{i}$ model}

One of the possibilities is to describe a tribological process using thermodynamic relationships [15]. Then, $\mathrm{f}(y)$ can be work done on 
a tribological system during test, $\mathrm{L}$ and $\phi(y)$ can be internal energy change $\Delta \mathrm{u}$. Then, equation 1 is as follows:

$$
\alpha_{i}=\left[\left(L-L_{o}\right) /\left(\Delta u-\Delta u_{o}\right)\right] x(d \Delta u / d L)
$$

$\mathrm{L}=\mu \mathrm{Pvt}$; where $\mu$ - friction coefficient; $\mathrm{v}$ - speed; $\mathrm{t}$ - time; $\mathrm{P}-$ applied load (test result); and $\mathrm{L}_{0}=$ $\mathrm{f}(a), \Delta \mathrm{u}_{0}=\phi(a)$ are respectively work done on the system and internal energy change, constant for the tested lubricant/reagent.

On the other hand, it is difficult to define relationship $\Delta \mathrm{u}=\phi(\mathrm{P})$, because the dependence between $\Delta \mathrm{u}$ and the applied load $\mathrm{P}$ is not linear. On the basis of the first law of thermodynamics, $L$ can be expressed as a function of $\Delta \mathrm{u}$ :

$$
L=Q+\Delta u
$$

Q is energy dissipated by system during a tribological process; mainly it is a dissipated heat, which in relation to tribological process can be described by the following dependence:

$$
\begin{gathered}
Q=c_{h}\left(T-T_{o t}\right) \\
T=T_{b}+A P^{0,5}
\end{gathered}
$$

where $c_{h}$ is an average specific heat capacity, $\mathrm{T}_{b}$ - temperature of lubricant out of the friction area, $\mathrm{T}_{o t}$ - temperature of environment, $\mathrm{A}$ - constant coefficient.

Assuming that both the average specific heat capacity and $\mathrm{T}_{b}$ are constant for different oils, $\mathrm{Q}$ can be expressed as $\mathrm{Q}=\mathrm{A}_{1} \mathrm{P}^{0.5}$, where $\mathrm{A}_{1}$ is a constant coefficient. Accordingly, the $\Delta \mathrm{u}$ can be presented as:

$$
\Delta u=L-Q=\mu v t P-A_{1} P^{0.5}
$$

In this case $\mathrm{C}$ takes the form:

$$
\begin{gathered}
C=(d \Delta u / d L) /\left(\Delta u-\Delta u_{o}\right) \\
C=\left[\left(\mu v t d P-A_{1} d P^{0.5}\right) / \mu v t d P\right] / \\
\left(\mu v t P-A_{1} P^{0.5}-\Delta u_{o}\right)
\end{gathered}
$$

$\mathrm{C}$ is a function of $\mathrm{P}$ and, referring this function to Cauchy's theorem, $\mathrm{P}$ can be the only one variable in functions $\mathrm{f}(y)$ and $\phi(y)$. The dependence (17) can be more complex in case when $\mu$ and $A_{1}$ are not fixed and they are functions of $\mathrm{P}$.

Roberto C Dante found that the $\alpha_{i}$ model can be related to thermodynamics of irreversible processes. Most recently, the results have been reported by Dante, Kajdas and Kulczycki [7]. In the $\alpha_{i}$ model, there are two independent variables: the applied load $\mathrm{P}$ and the chemical structure of a lubricant (CSL), i.e. the reagent as described and discussed by Kajdas and Kulczycki in the paper [8]. The latter variable was often given qualitatively, but from the tribochemical process point of view it was necessary to express it as a quantitative variable by a mathematical dependence. In this model it was assumed that CSL is expressed by $\alpha_{i}$, which is a measure of lubricant ability to create a boundary layer. It was also assumed that the value of $\alpha_{i}$ is a linear function of critical load P, i.e. the load is causing destruction of the generated boundary layer. The term $\alpha_{i}$, which is correlated to CSL, assumes the forms that drive the thermodynamics of irreversible processes (TIP). Dante et al. found [7] that equation 10 formally resembles the relation between entropy production $\sigma$, the force $\mathrm{X}$, and the corresponding flux $\mathrm{J}_{x}$ :

$$
\sigma=J_{x} X
$$

The relation between $\alpha_{i}$ and $\sigma$ is proposed to be the following:

$$
\sigma_{c \tau}=z d \alpha_{i} / d t
$$

where the sub-indexes $c$ and $\tau$ stay for chemical reaction and shear stress, respectively, while $\mathrm{z}$ is a proportionality factor. Since there is a relation between the chemical structure of lubricant and entropy, for analogy, it was assumed that $\alpha_{i}$ is directly linked to the entropy related to chemical reactions induced by friction. 


\section{Hypothesis on $\mathbf{E}_{a}$ of heteroge- neous and tribo-catalysis as well as mechanochemical reactions}

Chemical reactivity and the mechanical work done on surfaces seem to be clearly connected to the material surface states. These reactions (known as tribochemical reactions) proceed much faster under friction than thermochemical ones for the same reactants. In recent work Kajdas and Kulczycki [8] introduced a novel concept that the action of solid heterogeneous catalysts could be studied by comparing it to the action of mechanically activated solids in tribocatalysis. They proposed that for catalyzed heterogeneous reactions the same $\mathrm{E}_{a}$ (real activation energy for the non-catalyzed reaction) is needed to initiate the process, and that the difference between $\mathrm{E}_{a}$ and apparent activation energy $\left(\mathrm{E}_{a p p}\right)$ can be explained by adding the energy emitted by catalyst $\left(\mathrm{E}_{c}\right)$.

The work of Kajdas and Kulczycki [16] hypothesized that for standard and catalyzed heterogeneous reactions the same $\mathrm{E}_{a}$ (real activation energy) is needed to initiate the reaction processes. It has been proposed that an energy introduced into tribological system as mechanical work done on the solid material, is accumulated in this material and then emitted as electrons and/or photons to the space, in which the reactions take place. The developed model includes the specific $\gamma$ angle, at which the stream of energy $\mathrm{e}_{\gamma}$, in the range of 3 to $5 \mathrm{eV}$, is emitted and the reaction can be initiated. This idea results from $\alpha_{i}$ model but it should be confirmed experimentally. As it results from the work of Prevenslik [17], during a tribological processes the energy introduced to the tribological system is first stored in molecules, being harmonic oscillators, and then emitted by UV photons. This phenomenon can take place in a liquid (lubricant) or in a solid phase on the surface of lubricated elements or/and in the material of solid in dislocations. The possibilities and mechanism of energy storage is the key problem for tribochemistry, mechanochemistry and catalysis.

The distribution of the energy emitted by the solid body (e.g. by the catalyst) is controlled by the angle of emission:

$$
e_{\gamma}=e_{0} \cos \gamma
$$

where $\gamma$ is a function of $\mathrm{P}$ (loading) and depends on the stream of energy introduced into the tribological system, $\mathrm{e}_{0}$ is the density of the energy flux in the perpendicular direction to the solid body surface [16].

Triboemitted electrons, which make the major part of the charged-tribo-particles emission stream from insulators and semiconductors, are known to be related to exo-emission (including chemical exoemission and fractoemission), and triboemitted electrons have been postulated as a key factor in the initiation and control of tribochemical reactions [18-25].

\section{Experimental confirmation of the above hypothesis}

Several results of mechanochemical experiments, described in a number of works, can also be applied to further confirm the hypothesis resulting from $\alpha_{i}$ model. At this point, it should be underlined that there are differences between mechanochemical reactions and heterogeneous catalysis and tribocatalysis. During mechanochemical reactions mechanical energy is introduced into reagents, and - in case of heterogeneous catalysis as well as tribocatalysis mechanical energy - is introduced into the catalyst. However, mechanical action on solids involves the same or similar effects in case of mechanochemistry and catalysis.

For example, the hypothesis may be confirmed by the results presented by Stainike and Tkacova [26]. They investigated the reactions of solids: quartz, periclase and zinc ferrite, under mechanical treatment. They have experimentally found:

- significant decrease of activation energy Ea calculated using Arrhenius equation, of quartz: cristobalin transformation as a result of mechanical treatment of reactants,

- influence of mechanical energy stream on the rate of reaction. 
Both conclusions are in a good agreement with those found using $\alpha_{i}$ model:

- Mechanical treatment enables reactants to reach the activation energy at ambient temperature, much lower than that resulted from Arrhenius equation.

- Reaction rate constant is a function of the flux of energy, mechanically introduced into the reaction system:

$$
k=k\left\{A\left[\exp \left(E_{a} / R T\right)\right], \cos (\gamma), J\right\}
$$

where $\mathrm{T}$ is an ambient temperature of reaction mixture, $\mathrm{J}$ is the flux of energy mechanically introduced into the reaction system, $\cos (\gamma)$ is the angle of emission of electrons/photons of the energy adequate to initiate the reaction.

The hypothesis based on $\alpha_{i}$ model assumes the mechanical energy to be accumulated in the solid. Stainike and Tkacova [26] concluded that mechanically introduced energy is accumulated preferentially in the layers adjacent to the surface, due to cyclic microplastic deformations.

Very similar conclusions have been reported by Wieczorek-Ciurowa [27] in terms of mechanochemical synthesis, including mechanical alloying together with reactive milling, which follows the mechanical activation of reagents. It is emphasized that in the processes of plastic deformation/fracture and friction during ball collisions, the impact energy is converted into other forms of energy. These induce structural defects, broken bonds and other forms of excessive energy, which cause that new active states of the substances are produced. Consequently, the chemical reactivity of the solids increases significantly.

It was empirically observed during tribological tests that for lube oils containing additives of different chemical structures the constant value of $\mathrm{C}$ was obtained, while $\mathrm{C}$ is a function of the variable y. Such a condition can be satisfied for particular values of $\mathrm{y}$, where $\mathrm{C}$ is described by the relation:

$$
C=A[\exp (-a y)]\left[\left(e_{0}\right) \cos (b y+d)\right] / t \varepsilon^{2}
$$

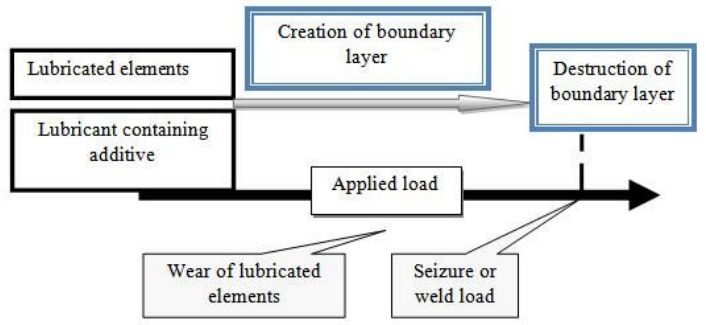

Fig. 2. Steps of boundary layer destruction leading to the seizure or weld processes.

where $\mathrm{y}=\mathrm{P}$ or $\mathrm{T}, \mathrm{e}_{0}$ is the energy flux in a direction perpendicular to the surface, $\varepsilon$ is the stream of mechanical energy stored in the solid, $t$ is time of the mechanical energy input, A, a, b and d are fixed. The reaction rate constant expressed by Arrhenius and Eyring law:

$$
k=A \exp (-E a / R T)
$$

points out that the only way to describe the catalytic effect is decreasing $\mathrm{E}_{a}$ value.

The Kar-Liang equation:

$$
k=\left(k_{0} T / h\right) \exp \left(-\Delta G^{\#}+\varepsilon\right) / R T
$$

where $\varepsilon$ is the mechanical energy, together with the dependence resulting from $\alpha_{i}$ model (Kajdas, Kulczycki):

$$
k=A \exp \left[-E_{a} /(R T+\varepsilon)\right] e_{0} \cos (b \varepsilon+d) / \varepsilon
$$

i.e.

$$
k=C t \varepsilon
$$

leads to the conclusion, that mechanical energy can be stored in the material of a catalyst and then emitted through the catalyst surface to the reaction zone, and this is the additional portion of energy supplied to the reactants. The activation energy as well as free energy of activation are constant for a given reaction and independent of the presence of a catalyst.

Arrhenius and Eyring equation lead to the conclusion that mechanical energy reduces both activation energy as well as free energy of activation.

The idea, illustrated by Fig. 3, leads to the opposite conclusion, that during a catalytic process 


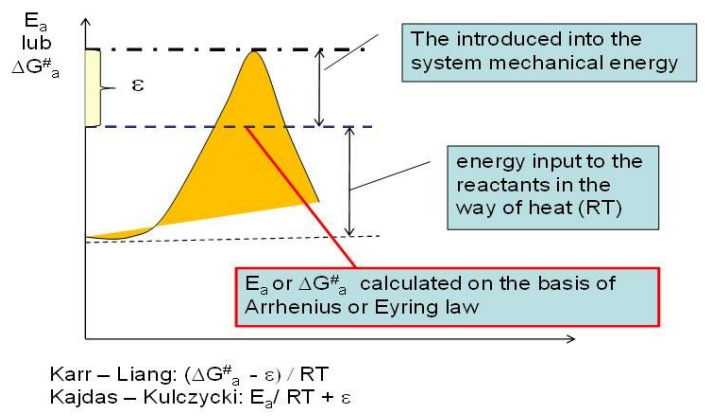

Fig. 3. The comparison of the Kar-Liang and Kulczycki-Kajdas ideas of the influence of mechanical energy on the reaction rate constant.

the value of activation energy as well as free energy of activation are constant for a given reaction and independent of the presence of a catalyst. Mechanical energy introduced into the system (into catalyst material) is the additional portion of energy, which enables reaching the activation energy at lower temperature. Kar-Liang equation describes this effect as:

$$
\left(-\Delta G_{a}^{\#}+\varepsilon\right) / R T
$$

while Kajdas and Kulczycki describe it as:

$$
E_{a} /(R T+\varepsilon)
$$

where $E_{a}$ and $-\Delta G_{a}^{\#}$ are activation energy and free energy of activation obtained for reaction without a catalyst.

Both equations, formulated on the basis of experimental data, lead to similar conclusion that mechanical energy introduced into a solid body during tribological process increases the reaction rate constant. The difference between Kar-Liang equation and those formulated by Kajdas and Kulczycki is in the exponential and in trigonometric part present in the equation formulated by Kajdas and Kulczycki. Kar and Liang formulated their equation on the basis of thermodynamics of reversible processes, while Kajdas and Kulczycki on the basis of thermodynamics of irreversible processes. This led to the conclusion that:

- Tribological processes, which take place under medium conditions, should be treated as reversible, while the processes under critical conditions (seizure, weld) as irreversible.

- Experimentally confirmed description (by Kar-Liang equation) of reversible tribological processes points out that $\cos \gamma=1$ and consequently $\gamma=0$.

- Experimentally confirmed description (by Kajdas and Kulczycki equation) of irreversible tribological processes points out that $\cos \gamma<1$ and consequently $\gamma>0$.

The above conclusions should be confirmed experimentally.

\section{Conclusions}

This paper demonstrates that mechanoactivation of solid body triggers heterogenous catalysis, which is controlled by low-energy triboelectrons in case of tribochemical reactions. Two areas of conditions of tribological processes have been taken into account: one connected with thermodynamically reversible processes and the other one connected with thermodynamically irreversible processes. The reversible processes take place when friction does not cause intensive wear. The rate constant of reactions occurring under conditions suitable for reversible processes was described by Kar-Liang equation and for irreversible ones by the equation formulated by Kajdas and Kulczycki. The main difference between the descriptions of reversible and irreversible tribological processes corresponds to trigonometric part of equation resulting from $\alpha_{i}$ model: in case of reversible processes $\cos \gamma=1$, and in case of irreversible processes $\cos \gamma<1$. The reviewed data confirm the anisotropy of angular distribution of exoelectrons emitted from perturbed surfaces. The model discussed in this paper provides a new approach to the activation energy and leads to better understanding of heterogeneous catalysis including tribocatalysis. Although the discussed anisotropy of electron emission relates to metalinsulator-metal thin film sandwich structures, we consider this fact as a first evidence for the proposed model, which predicts such triboelectron 
distribution. These conclusions should be verified experimentally.

\section{Acknowledgements}

Authors acknowledge Bartosz Gawron $\mathrm{PhD}$ for discussion and important comments to the paper.

\section{References}

[1] TAKaCs L., Nanostruct. Mater., 2 (1993), 241.

[2] Takacs L., Pollak H., Dlamini H., International Conference on the Applications of the Mossbauer Effect: ICAME 95, Italian Physical Society, Rimini (Italy), Bologna, 1996, p. 149.

[3] Balaż P., Choi W.-S., Fabian M., Godocikova E., Acta Montan. Slovaca, 11 (2006), 122.

[4] Grinfield M.A., Segletes S.B., ARL-TR-5309, U.S. Army Research Laboratory, Aberdeen Proving Ground, September, 2010.

[5] Kar P., Wang K., Liang H., Electrochem. Solid St., 11 (2008), 13.

[6] Kar P., Wang K., Liang H., Electrochim. Acta, 53 (2008), 5084.

[7] Dante R.C., Kajdas C.K., KulczycKi A., React. Kinet. Mech. Cat., 99 (2010), 37.

[8] Kajdas C.K., KulCZYCKI A., Mater. Sci.-Poland, 26 (2008), 787.

[9] KulCZYCKi A., Wear, 103 (1985), 67.

[10] KulcZYcKi A., Kajdas C., Lubr. Sci., 6 (1994), 161.

[11] Hrach R., MaY J., Phys. Status Solidi A, 4 (1971), 637.

[12] Gould R.D., Hoghart C.A., Phys. Status Solidi A, 41 (1977), 439.

[13] Hrach R., Czech. J. Phys. B, 23 (1973), 234.
[14] HraCh R., Thin Solid Films, 15 (1973), 65.

[15] Percec V., Keller A., Macromolecules, 23 (20) (1990) 4347.

[16] Kajdas C.K., Kulczycki A., KurzydŁowski K.J., Molina G.J., Mater. Sci.-Poland, 28 (2010), 523.

[17] Prevenslik T.V., Chinese J. Catal., 29 (11) (2008), 1073.

[18] Kajdas C., Furey M.J., Ritter A.L., Molina G.J., Lubr. Sci., 14 (2002), 223.

[19] Molina G.J., Kajdas C., Furey M.J., Proceedings of the Annual Meeting of the Adhesion Society, Savannah, Georgia, USA, February, 2009, p. 327.

[20] Kasemo B., Tornqvist E., Nordskold J.K., LundQVist B.I., Surf. Sci., 89 (1979), 554.

[21] Nienhaus H., Glass S., Surf. Sci., 600 (2006), 4285.

[22] Glass S., Nienhaus H., Phys. Rev. Lett., 93 (2004), 168302.

[23] Gergen B., Nienhaus H., Weiberger W.H., MCFrandL E.W., Science, 294 (2001), 2521.

[24] Boettcher A., Imbeck R., Morgante A., Ertl G., Phys. Rev. Lett., 65 (1990), 2035.

[25] CHEN Y.F., Surf. Sci., 519 (2002), 115.

[26] Stainike U., Tkacova K., J. Mater. Synth. Process., $8(3-4)(2000), 197$.

[27] Wieczorek-Ciurowa K., Dulian P., Nosal A., DomagaŁA J., J. Therm. Anal. Calorim., 101 (2010), 471.

[28] Marcus R.A., J. Chem. Phys., 24 (1956), 966.
Received 2014-04-18

Accepted 2014-07-06 